\title{
Liver fibrosis
}

\author{
Ramón Bataller ${ }^{1}$ and David A. Brenner ${ }^{2}$
}

'Liver Unit, Institut de Malalties Digestives i Metabòliques, Hospital Clinic, Institut d'Investigació Biomèdiques August Pi i Sunyer (IDIBAPS), Barcelona, Catalonia, Spain. ${ }^{2}$ Department of Medicine, Columbia University, New York, New York, USA.



Liver fibrosis is the excessive accumulation of extracellular matrix proteins including collagen that occurs in most types of chronic liver diseases. Advanced liver fibrosis results in cirrhosis, liver failure, and portal hypertension and often requires liver transplantation. Our knowledge of the cellular and molecular mechanisms of liver fibrosis has greatly advanced. Activated hepatic stellate cells, portal fibroblasts, and myofibroblasts of bone marrow origin have been identified as major collagen-producing cells in the injured liver. These cells are activated by fibrogenic cytokines such as TGF- $\beta 1$, angiotensin II, and leptin. Reversibility of advanced liver fibrosis in patients has been recently documented, which has stimulated researchers to develop antifibrotic drugs. Emerging antifibrotic therapies are aimed at inhibiting the accumulation of fibrogenic cells and/or preventing the deposition of extracellular matrix proteins. Although many therapeutic interventions are effective in experimental models of liver fibrosis, their efficacy and safety in humans is unknown. This review summarizes recent progress in the study of the pathogenesis and diagnosis of liver fibrosis and discusses current antifibrotic strategies.

\section{Historical perspective}

Liver fibrosis results from chronic damage to the liver in conjunction with the accumulation of ECM proteins, which is a characteristic of most types of chronic liver diseases (1). The main causes of liver fibrosis in industrialized countries include chronic HCV infection, alcohol abuse, and nonalcoholic steatohepatitis (NASH). The accumulation of ECM proteins distorts the hepatic architecture by forming a fibrous scar, and the subsequent development of nodules of regenerating hepatocytes defines cirrhosis. Cirrhosis produces hepatocellular dysfunction and increased intrahepatic resistance to blood flow, which result in hepatic insufficiency and portal hypertension, respectively (2).

Hepatic fibrosis was historically thought to be a passive and irreversible process due to the collapse of the hepatic parenchyma and its substitution with a collagen-rich tissue $(3,4)$. Currently, it is considered a model of the wound-healing response to chronic liver injury (5). Early clinical reports in the 1970s suggested that advanced liver fibrosis is potentially reversible (6). However, liver fibrosis received little attention until the 1980s, when hepatic stellate cells (HSCs), formerly known as lipocytes, Ito cells, or perisinusoidal cells, were identified as the main collagen-producing cells in the liver (7). This cell type, first described by von Kupffer in 1876, undergoes a dramatic phenotypic activation in chronic liver diseases with the acquisition of fibrogenic properties (8). Methods to obtain HSCs from both rodent and human livers were rapidly standardized in the 1980s $(9,10)$, and prolonged culture of HSCs on plastic was widely accepted as a model for the study of activated HSCs (11). Key signals that modulate HSCs' fibrogenic actions were delineated (12). Experimental models for studying liver fibrogenesis in rats and in transgenic mice were developed, which corroborated the cell culture studies and led to the identification of key fibrogenic mediators (13). Besides HSCs, portal myofibroblasts and cells of bone marrow ori-

Nonstandard abbreviations used: CTLA, cytotoxic T lymphocyte antigen; HSC, hepatic stellate cell; NASH, nonalcoholic steatohepatitis; PBC, primary biliary cirrhosis; TIMP-1, tissue inhibitor of metalloproteinase type 1.

Conflict of interest: The authors have declared that no conflict of interest exists.

Citation for this article: J. Clin. Invest. 115:209-218 (2005).

doi:10.1172/JCI200524282. gin have been recently shown to exhibit fibrogenic potential $(14,15)$. At the clinical level, the natural history of liver fibrosis, from early changes to liver cirrhosis, was delineated in patients with chronic HCV infection $(16,17)$. Rapid and slower fibrosers were identified, and genetic and environmental factors influencing fibrosis progression were partially uncovered (18). Since the demonstration, in the 1990s, that even advanced liver fibrosis is reversible, researchers have been stimulated to identify antifibrotic therapies (19). Biotechnology and pharmaceutical companies are increasingly interested in developing antifibrotic programs, and clinical trials are currently underway. However, the most effective therapy for treating hepatic fibrosis to date is still to remove the causative agent (20). A number of drugs are able to reduce the accumulation of scar tissue in experimental models of chronic liver injury. Renin-angiotensin system blockers and antioxidants are the most promising drugs, although their efficacy has not been tested in humans. Lack of clinical trials is due to the requirement of long follow-up studies and to the fact that liver biopsy, an invasive procedure, is still the gold-standard method for detecting changes in liver fibrosis. The current effort to develop noninvasive markers to assess liver fibrosis is expected to facilitate the design of clinical trials.

Recently, NASH has been recognized as a major cause of liver fibrosis (21). First described by Ludwig et al., it is considered part of the spectrum of nonalcoholic fatty liver diseases (22). These range from steatosis to cirrhosis and can eventually lead to hepatocellular carcinoma. NASH is a component of the metabolic syndrome, which is characterized by obesity, type 2 diabetes mellitus, and dyslipidemia, with insulin resistance as a common feature. As the prevalence of obesity is rapidly increasing, a rise in the prevalence of NASH is anticipated.

This review outlines recent progress in the pathogenesis, diagnosis, and treatment of liver fibrosis, summarizes recent data on the mechanisms leading to fibrosis resolution, and discusses future prospects aimed at developing effective antifibrotic therapies.

\section{Natural history and diagnosis}

The onset of liver fibrosis is usually insidious, and most of the related morbidity and mortality occur after the development of cirrhosis (16). In the majority of patients, progression to cirrhosis 


\section{Table 1}

Genetic and nongenetic factors associated with fibrosis progression in different types of chronic liver diseases

\begin{tabular}{|c|c|c|c|}
\hline Type of liver disease & Candidate genes & Candidate genes (full name) & Nongenetic factors \\
\hline \multirow[t]{9}{*}{ Chronic HCV infection } & HFE & Hereditary hemochromatosis gene & Alcohol intake \\
\hline & Angiotensinogen & Angiotensinogen & Coinfection HIV and/or hepatitis B virus \\
\hline & TGF- $\beta 1$ & Transforming growth factor $\beta 1$ & Age at time of acute infection \\
\hline & $T N F-\alpha$ & Tumor necrosis factor $\alpha$ & Liver transplantation \\
\hline & $A p o E$ & Apolipoprotein E & Diabetes mellitus \\
\hline & MEH & Microsomal epoxide hydroxylase & No response to therapy \\
\hline & $M C P-1$ & Monocyte chemotactic protein type 1 & \\
\hline & $M C P-2$ & Monocyte chemotactic protein type 2 & \\
\hline & Factor $\mathrm{V}$ & Factor V (Leiden) & \\
\hline \multirow[t]{9}{*}{ Alcohol-induced } & $I L-10$ & Interleukin 10 & Alcohol intake \\
\hline & $I L-1 \beta$ & Interleukin $1 \beta$ & Episodes of alcoholic hepatitis \\
\hline & $A D H$ & Alcohol dehydrogenase & \\
\hline & $A L D H$ & Aldehyde dehydrogenase & \\
\hline & CYP2E1 & cytochrome P450, family 2 , subfamily e, polypeptide 1 & \\
\hline & $T N F-\alpha$ & Tumor necrosis factor $\alpha$ & \\
\hline & CTLA-4 & Cytotoxic T lymphocyte antigen type 4 & \\
\hline & TAP2 & Transporter-associated antigen-processing type 2 & \\
\hline & MnSOD & Manganese superoxide dismutase & \\
\hline \multirow[t]{4}{*}{$\mathrm{NASH}$} & HFE & Hereditary hemochromatosis gene & Age \\
\hline & Angiotensinogen & Angiotensinogen & Severity of obesity \\
\hline & TGF- $\beta 1$ & Transforming growth factor $\beta 1$ & Diabetes mellitus \\
\hline & & & Hypertriglyceridemia \\
\hline \multirow[t]{3}{*}{ PBC } & $I L-1 \beta$ & Interleukin $1 \beta$ & \\
\hline & $T N F-\alpha$ & Tumor necrosis factor $\alpha$ & \\
\hline & $A p o E$ & Apolipoprotein E & \\
\hline Autoimmune hepatitis & $H L A-/ /$ & Human leukocyte antigen type II haplotypes & $\begin{array}{l}\text { Type II autoimmune hepatitis } \\
\text { No response to therapy }\end{array}$ \\
\hline
\end{tabular}

occurs after an interval of 15-20 years. Major clinical complications of cirrhosis include ascites, renal failure, hepatic encephalopathy, and variceal bleeding. Patients with cirrhosis can remain free of major complications for several years (compensated cirrhosis). Decompensated cirrhosis is associated with short survival, and liver transplantation is often indicated as the only effective therapy (23). Cirrhosis is also a risk factor for developing hepatocellular carcinoma. Liver fibrosis progresses rapidly to cirrhosis in several clinical settings, including repeated episodes of severe acute alcoholic hepatitis, subfulminant hepatitis, and fibrosing cholestasis in patients with HCV reinfection after liver transplantation (24). The natural history of liver fibrosis is influenced by both genetic and environmental factors (Table 1). Epidemiological studies have identified polymorphisms in a number of candidate genes that may influence the progression of liver fibrosis in humans (18). These genetic factors may explain the broad spectrum of responses to the same etiological agent found in patients with chronic liver diseases. However, some studies have yielded contradictory results due to poor study design, and further research is required to clarify the actual role of genetic variants in liver fibrosis.

Liver biopsy is considered the gold-standard method for the assessment of liver fibrosis (25). Histologic examination is useful in identifying the underlying cause of liver disease and assessing the necroinflammatory grade and the stage of fibrosis. Fibrosis stage is assessed by using scales such as Metavir (stages I-IV) and Ishak score (stages I-V). Specific staining of ECM proteins (e.g., with Sirius red) can be used to quantify the degree of fibrosis, using computer-guided morphometric analysis. Liver biopsy is an invasive procedure, with pain and major complications occurring in $40 \%$ and $0.5 \%$ of patients, respectively (26). Sampling error can occur, especially when small biopsies are analyzed. Histologic examination is prone to intra- and interobserver variation and does not predict disease progression (27). Therefore, there is a need for reliable, simple, and noninvasive methods for assessing liver fibrosis. Scores that include routine laboratory tests, such as platelet count, aminotransferase serum levels, prothrombin time, and serum levels of acute phase proteins have been proposed $(28,29)$. Serum levels of proteins directly related to the hepatic fibrogenic process are also used as surrogate markers of liver fibrosis (30), including N-terminal propeptide of type III collagen, hyaluronic acid, tissue inhibitor of metalloproteinase type 1 (TIMP-1), and YKL-40. Although these scores are useful in detecting advanced fibrosis (cirrhosis) in patients, as well as minimal or no fibrosis, they are not effective for differentiating intermediate grades of fibrosis. Also, fibrosis-specific markers may reflect fibrogenesis in other organs (i.e., pancreatic fibrosis in alcoholic patients). Finally, hepatic fibrosis can be estimated by imaging techniques. Ultrasonography, computed tomography, and MRI can detect changes in the hepatic parenchyma due to moderate to severe fibrosis (31). Due to its low cost, ultrasonography is an appealing technique. It is able to detect liver cirrhosis based on changes in liver echogenicity and nodularity as well as signs of portal hypertension. However, ultrasound is highly operatordependent, and the presence of increased liver echogenicity does not reliably differentiate hepatic steatosis from fibrosis. Noninvasive methods currently in development include blood protein 


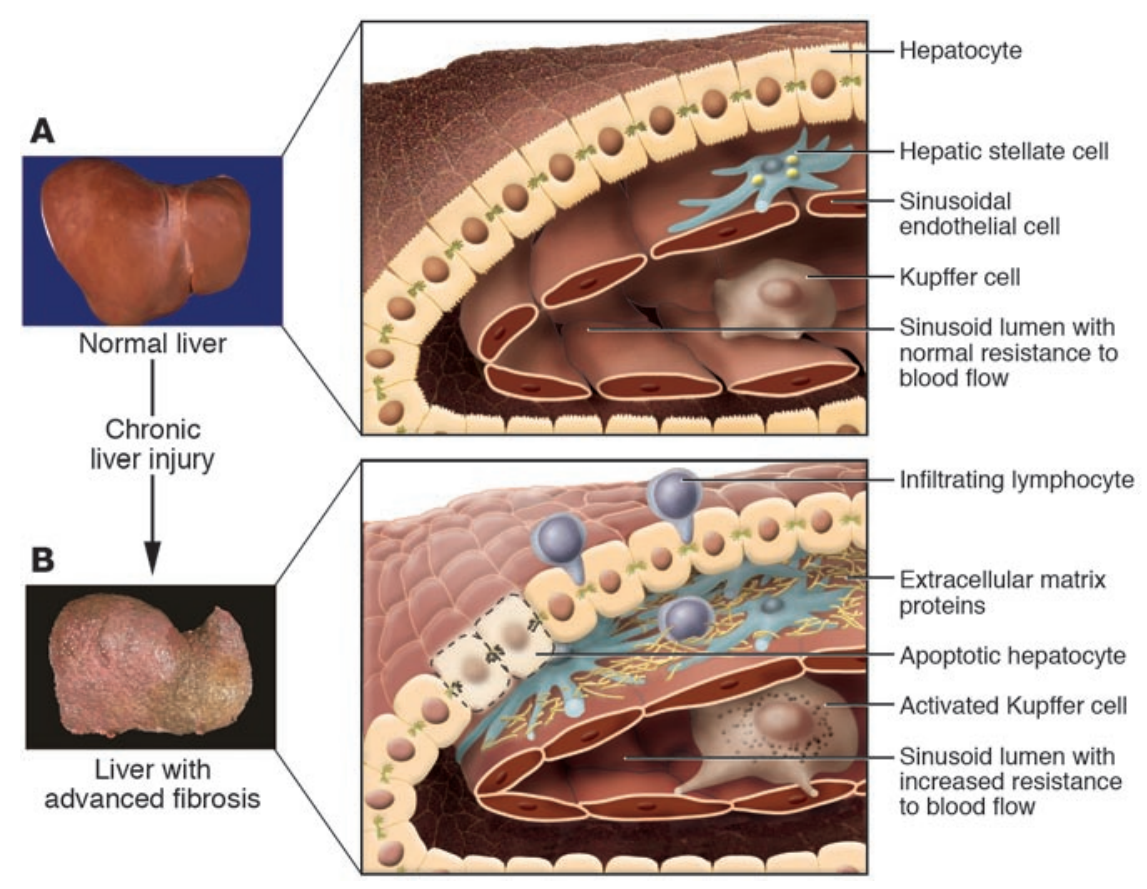

\section{Figure 1}

Changes in the hepatic architecture (A) associated with advanced hepatic fibrosis (B). Following chronic liver injury, inflammatory lymphocytes infiltrate the hepatic parenchyma. Some hepatocytes undergo apoptosis, and Kupffer cells activate, releasing fibrogenic mediators. HSCs proliferate and undergo a dramatic phenotypical activation, secreting large amounts of extracellular matrix proteins. Sinusoidal endothelial cells lose their fenestrations, and the tonic contraction of HSCs causes increased resistance to blood flow in the hepatic sinusoid. Figure modified with permission from Science \& Medicine (S28). profiling using proteomic technology and new clinical glycomics technology, which is based on DNA sequencer/fragment analyzers able to generate profiles of serum protein $N$-glycans (32). As the technology becomes validated, the noninvasive diagnosis of liver disease may become routine clinical practice.

\section{Pathogenesis of liver fibrosis}

Hepatic fibrosis is the result of the wound-healing response of the liver to repeated injury (1) (Figure 1). After an acute liver injury (e.g., viral hepatitis), parenchymal cells regenerate and replace the necrotic or apoptotic cells. This process is associated with an inflammatory response and a limited deposition of ECM. If the hepatic injury persists, then eventually the liver regeneration fails, and hepatocytes are substituted with abundant ECM, including fibrillar collagen. The distribution of this fibrous material depends on the origin of the liver injury. In chronic viral hepatitis and chronic cholestatic disorders, the fibrotic tissue is initially located around portal tracts, while in alcohol-induced liver disease, it locates in pericentral and perisinusoidal areas (33). As fibrotic liver diseases advance, disease progression from collagen bands to bridging fibrosis to frank cirrhosis occurs.

Liver fibrosis is associated with major alterations in both the quantity and composition of ECM (34). In advanced stages, the liver contains approximately 6 times more ECM than normal, including collagens (I, III, and IV), fibronectin, undulin, elastin, laminin, hyaluronan, and proteoglycans. Accumulation of ECM results from both increased synthesis and decreased degradation (35). Decreased activity of ECM-removing MMPs is mainly due to an overexpression of their specific inhibitors (TIMPs).

HSCs are the main ECM-producing cells in the injured liver (36). In the normal liver, HSCs reside in the space of Disse and are the major storage sites of vitamin A. Following chronic injury, HSCs activate or transdifferentiate into myofibroblast-like cells, acquiring contractile, proinflammatory, and fibrogenic properties $(37,38)$ (Figure 2A). Activated HSCs migrate and accumulate at the sites of tissue repair, secreting large amounts of ECM and regulating ECM degradation. PDGF, mainly produced by Kupffer cells, is the predominant mitogen for activated HSCs. Collagen synthesis in HSCs is regulated at the transcriptional and posttranscriptional levels (39). Increased collagen mRNA stability mediates the increased collagen synthesis in activated HSCs. In these cells, posttranscriptional regulation of collagen is governed by sequences in the $3^{\prime}$ untranslated region via the RNA-binding protein $\alpha \mathrm{CP} 2$ as well as a stem-loop structure in the $5^{\prime}$ end of collagen mRNA (40). Interestingly, HSCs express a number of neuroendocrine markers (e.g., reelin, nestin, neurotrophins, synaptophysin, and glial-fibrillary acidic protein) and bear receptors for neurotransmitters (8, 41, 42).
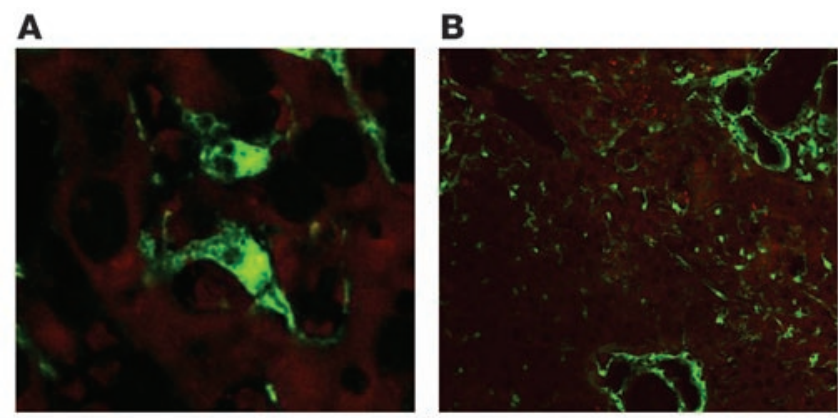

\section{Figure 2}

Expression of collagen $\alpha 1(\mathrm{I})$ in a model of cholestasis-induced liver fibrosis. Transgenic mice with green fluorescence protein reporter gene under the direction of the collagen $\alpha 1$ (I) promoter/enhancers were subjected to bile duct ligation for 2 weeks. (A) Collagen $\alpha 1$ (I) was markedly expressed by activated HSCs, but not hepatocytes, in the hepatic parenchyma. Magnification, $\times 200$. (B) Collagen $\alpha 1(\mathrm{I})$ is markedly expressed by myofibroblasts around proliferating bile ducts. HSCs proliferate to initiate collagen deposition in the hepatic parenchyma. Magnification, $\times 40$. 


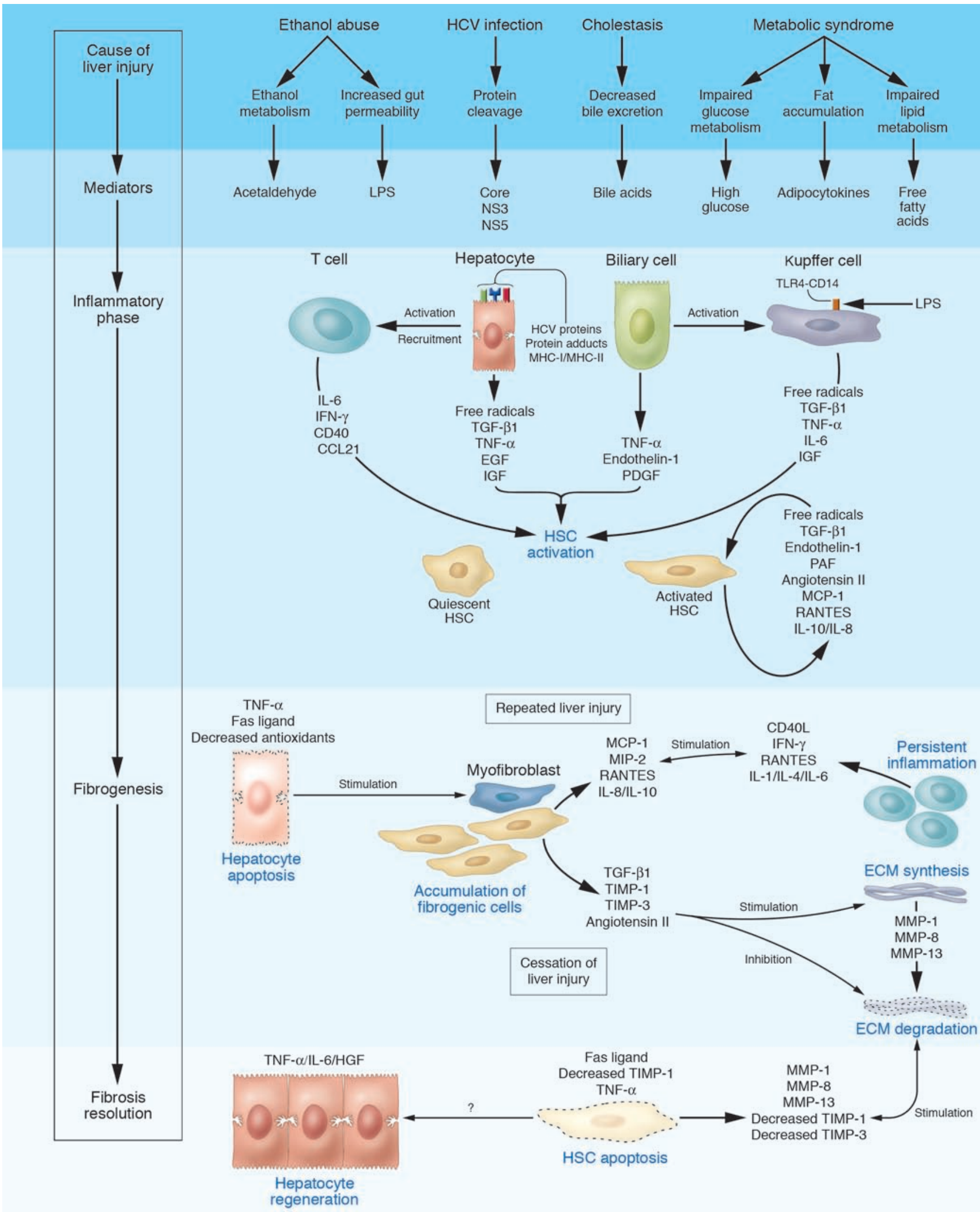


Quiescent HSCs express markers that are characteristic of adipocytes (PPAR $\gamma$, SREBP-1c, and leptin), while activated HSCs express myogenic markers ( $\alpha$ smooth muscle actin, $c-m y b$, and myocyte enhancer factor-2).

Hepatic cell types other than HSCs may also have fibrogenic potential. Myofibroblasts derived from small portal vessels proliferate around biliary tracts in cholestasis-induced liver fibrosis to initiate collagen deposition $(43,44)$ (Figure 2B). HSCs and portal myofibroblasts differ in specific cell markers and response to apoptotic stimuli (45). Culture of $\mathrm{CD} 34^{+} \mathrm{CD} 38^{-}$ hematopoietic stem cells with various growth factors has been shown to generate HSCs and myofibroblasts of bone marrow origin that infiltrate human livers undergoing tissue remodeling $(15,46)$. These data suggest that cells originating in bone marrow can be a source of fibrogenic cells in the injured liver. Other potential sources of fibrogenic cells (i.e., epithelial-mesenchymal transition and circulating fibrocytes) have not been demonstrated in the liver $(47,48)$. The relative importance of each cell type in liver fibrogenesis may depend on the origin of the liver injury. While HSCs are the main fibrogenic cell type in pericentral areas, portal myofibroblasts may predominate when liver injury occurs around portal tracts.

A complex interplay among different hepatic cell types takes place during hepatic fibrogenesis (Figure 3) (49). Hepatocytes are targets for most hepatotoxic agents, including hepatitis viruses, alcohol metabolites, and bile acids (50). Damaged hepatocytes release ROS and fibrogenic mediators and induce the recruitment of white blood cells by inflammatory cells. Apoptosis of damaged hepatocytes stimulates the fibrogenic actions of liver myofibroblasts (51). Inflammatory cells, either lymphocytes or polymorphonuclear cells, activate HSCs to secrete collagen (52). Activated HSCs secrete inflammatory chemokines, express cell adhesion molecules, and modulate the activation of lymphocytes (53). Therefore, a vicious circle in which inflammatory and fibrogenic cells stimulate each other is likely to occur (54). Fibrosis is influenced by different $\mathrm{T}$ helper subsets, the Th2 response being associated with more active fibrogenesis (55). Kupffer cells are resident macrophages that play a major role in liver inflammation by releasing ROS and cytokines $(56,57)$. In chronic cholestatic disorders (i.e., primary biliary cirrhosis [PBC] and primary sclerosis cholangitis), epithelial cells stimulate the accumulated portal myofibroblasts to initiate collagen deposition around damaged bile ducts (43). Finally, changes in the composition of the ECM can directly stimulate fibrogenesis. Type IV collagen, fibrinogen, and urokinase type plasminogen activator stimulate resident HSCs by activating latent cytokines such as TGF- $\beta 1$ (58). Fibrillar collagens can bind and stimulate HSCs via discoidin domain receptor DDR2 and integrins. Moreover, the altered ECM can serve as a reservoir for growth factors and MMPs (59).
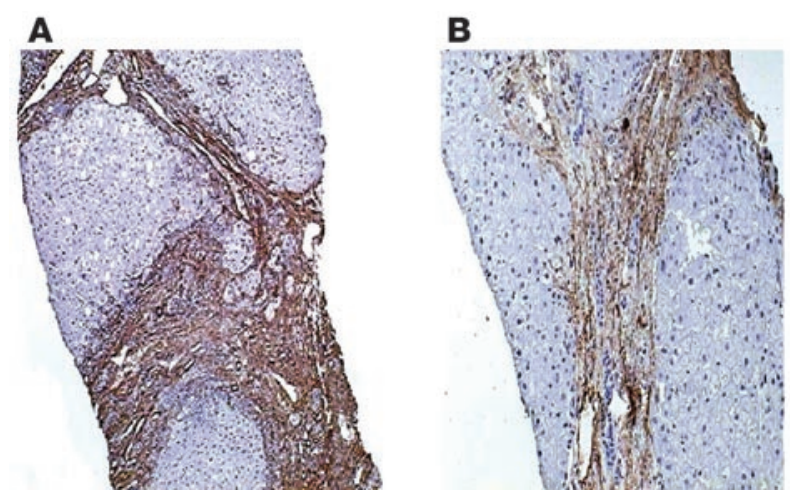

\section{Figure 4}

Reversibility of liver fibrosis in a patient with chronic hepatitis B virus infection after successful treatment with lamivudine. A decrease in smooth muscle actin immunostaining, a marker of fibrogenic myofibroblasts, can be seen in paired liver biopsies before $(\mathbf{A})$ and after (B) therapy. Dark brown granules represent areas stained for smooth muscle actin. Magnification, $\times 40$. Reproduced with permission from Journal of Hepatology (S2).

\section{Genetic studies in rodents and humans}

Extensive studies using models of hepatic fibrosis in transgenic mice have revealed key genes mediating liver fibrogenesis $(1,18)$. Genes regulating hepatocellular apoptosis and/or necrosis (e.g., Bcl-xL, Fas) influence the extent of hepatic damage and the subsequent fibrogenic response $(60,61)$. Genes regulating the inflammatory response to injury (e.g., IL-1 $\beta$, IL-6, IL-10, and IL-13, IFN- $\gamma$, SOCS-1, and osteopontin) determine the fibrogenic response to injury $(55,62-65)$. Genes mediating ROS generation (e.g., NADPH oxidase) regulate both inflammation and ECM deposition (66). Fibrogenic growth factors (e.g., TGF- $\beta 1$, FGF), vasoactive substances (angiotensin II, norepinephrine), and adipokines (leptin and adiponectin) are each required for the development of fibrosis (67-70). Finally, removal of excess collagen after cessation of liver injury is regulated by TIMP- 1 and TGF- $\beta 1(71,72)$.

Association genetic studies have investigated the role of gene polymorphisms in the progression of liver fibrosis in patients with chronic liver diseases (18). In alcoholic liver disease, candidate genes include genes encoding for alcohol-metabolizing enzymes and proteins involved in liver toxicity (73). Polymorphisms in genes encoding alcohol-dehydrogenase, aldehyde-dehydrogenase, and cytochrome P450 are involved in individual susceptibility to alcoholism, yet their role in the progression of liver disease remains controversial. Variations in genes encoding inflammatory mediators (e.g., TNF- $\alpha$, IL-1 $\beta$, Il-10, and cytotoxic T lymphocyte antigen-4 [CTLA-4]), the lipopolysaccharide receptor CD14, and antioxidants (e.g., superoxide dismutase) may influence the

\section{Figure 3}

Cellular mechanisms of liver fibrosis. Different types of hepatotoxic agents produce mediators that induce inflammatory actions in hepatic cell types. Damaged hepatocytes and biliary cells release inflammatory cytokines and soluble factors that activate Kupffer cells and stimulate the recruitment of activated T cells. This inflammatory milieu stimulates the activation of resident HSCs into fibrogenic myofibroblasts. Activated HSCs also secrete cytokines that perpetuate their activated state. If the liver injury persists, accumulation of activated HSCs and portal myofibroblasts occurs, synthesizing large amounts of ECM proteins and leading to tissue fibrosis. ECM degradation is inhibited by the actions of cytokines such as TIMPs. Apoptosis of damaged hepatocytes stimulates the fibrogenic actions of HSCs. If the cause of the liver injury is removed, fibrosis is resolved. This phase includes apoptosis of activated HSCs and regeneration of hepatocytes. Collagen is degraded by increased activity of MMPs induced by decreased TIMP expression. CCL21, C-C chemokine ligand 21; MCP-1, monocyte chemoattractant protein-1; MIP-2, macrophage inflammatory protein-2; NS3, HCV nonstructural protein 3; NS5, HCV nonstructural protein 5; PAF, platelet-activating factor. 
progression of alcohol-induced liver disease $(74,75)$. In chronic cholestatic disorders such as PBC, polymorphisms in IL-1 $\beta$, IL-1 receptor antagonists, and TNF- $\alpha$ genes are associated with faster disease progression (76). Some alleles of the apolipoprotein $\mathrm{E}$ gene influence the response to therapy of $\mathrm{PBC}$ with ursodeoxycholic acid, which suggests that genetic polymorphisms may predict therapeutic response (77). In HCV liver disease, genetic variations are involved in susceptibility to persistent $\mathrm{HCV}$ infection, response to antiviral therapy, and progression of liver disease (78). Polymorphisms in genes involved in the immune response to $\mathrm{HCV}$ infection (e.g., transporter associated with antigen processing 2 , mannose-binding lectin, and specific HLA-II alleles) and fibrogenic agonists (angiotensinogen and TGF- $\beta 1$ ) influence fibrosis progression (79-81). The fibrogenic effect of heterozygosity in the $\mathrm{C} 282 \mathrm{Y}$ mutation of the hemochromatosis gene in patients with chronic hepatitis $\mathrm{C}$ is controversial $(82,83)$. Finally, little is known about genetic factors and NASH (84), and polymorphisms in fibrogenic mediators such as angiotensinogen and TGF- $\beta 1$ may be associated with more severe liver disease.

\section{Key cytokines involved in liver fibrosis}

Cytokines regulating the inflammatory response to injury modulate hepatic fibrogenesis in vivo and in vitro (85). Monocyte chemotactic protein type 1 and RANTES stimulate fibrogenesis while IL-10 and IFN- $\gamma$ exert the opposite effect $(55,86)$. Among growth factors, TGF- $\beta 1$ appears to be a key mediator in human fibrogenesis (58). In HSCs, TGF- $\beta$ favors the transition to myofibroblast-like cells, stimulates the synthesis of ECM proteins, and inhibits their degradation. Strategies aimed at disrupting TGF- $\beta 1$ synthesis and/or signaling pathways markedly decreased fibrosis in experimental models (87). PDGF is the most potent mitogen for HSCs and is upregulated in the fibrotic liver (12); its inhibition attenuates experimental liver fibrogenesis (88).

Cytokines with vasoactive properties also regulate liver fibrogenesis. Vasodilator substances (e.g., nitric oxide, relaxin) exert antifibrotic effects while vasoconstrictors (e.g., norepinephrine, angiotensin II) have opposite effects $(67,89)$. Endothelin-1, a powerful vasoconstrictor, stimulates fibrogenesis through its type A receptor (90). Among vasoactive cytokines, angiotensin II seems to play a major role in liver fibrogenesis. Angiotensin II is the effector peptide of the renin-angiotensin system, which is a major regulator of arterial pressure homeostasis in humans. Key components of this system are locally expressed in chronically injured livers, and activated HSCs de novo generate angiotensin II $(91,92)$. Importantly, pharmacological and/or genetic ablation of the renin-angiotensin system markedly attenuates experimental liver fibrosis (70, 93-98). Angiotensin II induces hepatic inflammation and stimulates an array of fibrogenic actions in activated HSCs, including cell proliferation, cell migration, secretion of proinflammatory cytokines, and collagen synthesis $(66,99,100)$. These actions are largely mediated by ROS generated by a nonphagocytic form of NADPH oxidase. Unlike the phagocytic type, NADPH oxidases present in fibrogenic cell types are constitutively active, producing relatively low levels of ROS under basal conditions and generating higher levels of oxidants in response to cytokines, stimulating redox-sensitive intracellular pathways. NADPH oxidase also plays a key role in the inflammatory actions of Kupffer cells (101). Disruption of an active NADPH oxidase protects mice from developing severe liver injury following prolonged alcohol intake and/or bile duct ligation $(66,102)$.

Adipokines, which are cytokines mainly derived from the adipose tissue, regulate liver fibrogenesis. Leptin is required for HSC activation and fibrosis development $(103,104)$. In contrast, adiponectin markedly inhibits liver fibrogenesis in vitro and in vivo (69). The actions of these cytokines may explain why obesity influences fibrosis development in patients with chronic hepatitis C (105).

\section{Intracellular signaling pathways mediating liver fibrogenesis}

Data on intracellular pathways regulating liver fibrogenesis are mainly derived from studies using cultured HSCs, while understanding of their role in vivo is progressing through experimental fibrogenesis studies using knockout mice (106). Several mitogen-

\section{Table 2}

Main antifibrotic drugs in development for the treatment of liver fibrosis

\begin{tabular}{|c|c|c|c|c|}
\hline Agent & Main mechanism & $\begin{array}{c}\text { Antifibrotic effects } \\
\text { in HSCs }\end{array}$ & $\begin{array}{l}\text { Antifibrotic effects in } \\
\text { experimental fibrosis }\end{array}$ & $\begin{array}{l}\text { Antifibrotic effect } \\
\text { in humans }\end{array}$ \\
\hline Angiotensin inhibitors & Inhibits HSC activation & Consistent positive data & Consistent positive data & Retrospective study \\
\hline Colchicine & Inhibits inflammatory response & Limited data & Limited data & Discrepant results \\
\hline Corticosteroids & Inhibits inflammatory response & Limited data & Limited data & $\begin{array}{l}\text { Effective in } \\
\text { autoimmune hepatitis }\end{array}$ \\
\hline Endothelin inhibitors & Inhibits HSC function & Limited data & Limited data & Not tested \\
\hline Interferon- $\alpha$ & Inhibits HSC activation & Consistent positive data & Consistent positive data & Effective in chronic hepatitis C \\
\hline Interleukin 10 & Inhibits inflammatory response & Limited data & Consistent positive data & $\begin{array}{c}\text { Isolated reports } \\
\text { in chronic hepatitis C }\end{array}$ \\
\hline Pentoxifylline & Inhibits HSC activation & Consistent positive data & Consistent positive data & Not tested \\
\hline Phosphatidylcholine & Decreases oxidative stress & Limited data & Consistent positive data & $\begin{array}{l}\text { Not proven in } \\
\text { alcohol-induced fibrosis }\end{array}$ \\
\hline PPAR antagonists & Inhibits HSC activation & Consistent data & Consistent positive data & Isolated reports in NASH \\
\hline S-adenosyl-methionine & Antioxidant & Limited data & Not tested & $\begin{array}{l}\text { Effective in } \\
\text { alcohol-induced fibrosis }\end{array}$ \\
\hline Sho-saiko-to & Antioxidant & Consistent positive data & Consistent positive data & $\begin{array}{l}\text { Isolated reports in } \\
\text { chronic hepatitis C }\end{array}$ \\
\hline TGF- $\beta 1$ inhibitors & Inhibits HSC activation and function & Consistent positive data & Consistent positive data & Not tested \\
\hline Tocopherol & Antioxidant & Consistent positive data & Limited data & Isolated reports in NASH \\
\hline
\end{tabular}


activated protein kinases modulate major fibrogenic actions of HSCs. Extracellular-regulated kinase, which is stimulated in experimentally induced liver injury, mediates proliferation and migration of HSCs (107). In contrast, c-Jun N-terminal kinase regulates apoptosis of hepatocytes as well as the secretion of inflammatory cytokines by cultured HSCs $(66,108,109)$. The focal adhesion kinase PI3K-Akt-signaling pathway mediates agonist-induced fibrogenic actions in HSCs (107). The TGF- $\beta 1$-activated Smadsignaling pathway stimulates experimental hepatic fibrosis and is a potential target for therapy $(110,111)$. The PPAR pathway regulates HSC activation and experimental liver fibrosis. PPAR- $\gamma$ ligands inhibit the fibrogenic actions in HSCs and attenuate liver fibrosis in vivo $(112,113)$. NF-KB may have an inhibitory action on liver fibrosis $(114,115)$. Other transcription factors are involved in HSC activation and may participate in liver fibrogenesis (116). Recent studies suggest a role for intracellular pathways signaled by Toll-like receptors and $\beta$-cathepsin $(117,118)$.

\section{Pathogenesis of fibrosis in different liver diseases}

The pathogenesis of liver fibrosis depends on the underlying etiology. In alcohol-induced liver disease, alcohol alters the population of gut bacteria and inhibits intestinal motility, resulting in an overgrowth of Gram-negative flora. Lipopolysaccharide is elevated in portal blood and activates Kupffer cells through the CD14/Toll-like receptor-4 complex to produce ROS via NADPH oxidase (101). Oxidants activate Kupffer cell NF- $\kappa B$, causing an increase in TNF- $\alpha$ production. TNF- $\alpha$ induces neutrophil infiltration and stimulates mitochondrial oxidant production in hepatocytes, which are sensitized to undergo apoptosis. Acetaldehyde, the major alcohol metabolism product, and ROS activate HSCs and stimulate inflammatory and fibrogenic signals (119). The pathogenesis of HCV-induced liver fibrosis is poorly understood due to the lack of a rodent model of persistent HCV infection (78). HCV escapes surveillance of the HLA-II-directed immune response and infects hepatocytes, causing oxidative stress and inducing the recruitment of inflammatory cells. Both factors lead to HSC activation and collagen deposition. Moreover, several HCV proteins directly stimulate the inflammatory and fibrogenic actions of HSCs (120). In chronic cholestatic disorders such as PBC, T lymphocytes and cytokines mediate persistent bile duct damage (14). Biliary cells secrete fibrogenic mediators activating neighboring portal myofibroblasts to secrete ECM. Eventually, perisinusoidal HSCs become activated, and fibrotic bands develop. The pathogenesis of liver fibrosis due to NASH is poorly understood. Obesity, type 2 diabetes mellitus, and dyslipidemia are the most common associated conditions (121). A 2-hit model has been proposed: hyperglycemia and insulin resistance lead to elevated serum levels of free fatty acids, resulting in hepatic steatosis. In the second hit, oxidative stress and proinflammatory cytokines promote hepatocyte apoptosis and the recruitment of inflammatory cells, leading to progressive fibrosis.

\section{Is liver fibrosis reversible?}

In contrast with the traditional view that cirrhosis is an irreversible disease, recent evidence indicates that even advanced fibrosis is reversible (122). In experimentally induced fibrosis, cessation of liver injury results in fibrosis regression (123). In humans, spontaneous resolution of liver fibrosis can occur after successful treatment of the underlying disease. This observation has been described in patients with iron and copper overload, alcohol-induced liver injury, chronic hepatitis C, B, and D, hemochromatosis, secondary biliary cirrhosis, NASH, and autoimmune hepatitis $(19,122,124,125$, S1, S2) (Figure 4). It may take years for significant regression to be achieved; the time varies depending on the underlying cause of the liver disease and its severity. Chronic $\mathrm{HCV}$ infection is the most extensively studied condition, and therapy (IFN- $\alpha$ plus ribavirin) with viral clearance results in fibrosis improvement. Importantly, nearly half of patients with cirrhosis exhibit reversal to a significant degree (90). Whether this beneficial effect is associated with improvements in long-term clinical outcome, including decreased portal hypertension, is unknown.

Increased collagenolytic activity is a major mechanism of fibrosis resolution (122). Fibrillar collagens (I and III) are degraded by interstitial MMPs (MMP-1, -8 , and -13 in humans and MMP-13 in rodents). During fibrosis resolution, MMP activity increases due to a rapid decrease in the expression of TIMP-1. Partial degradation of fibrillar collagen occurs, and the altered interaction between activated HSCs and ECM favors apoptosis (123). Removal of activated HSCs by apoptosis precedes fibrosis resolution. Stimulation of death receptors in activated HSCs and a decrease in survival factors, including TIMP-1, can precipitate HSC apoptosis (S3).

Several questions remain unanswered: Can we pharmacologically accelerate fibrosis resolution in humans? Can a fibrotic liver completely regress to a normal liver? Does fibrosis reverse similarly in all types of liver diseases? Although isolated cases of complete fibrosis resolution have been reported, it is conceivable that some degree of fibrosis cannot be removed (S4). Resolution may be limited by ECM cross-linking and a failure of activated HSCs to undergo apoptosis.

\section{Therapeutic approaches to the treatment of liver fibrosis}

There is no standard treatment for liver fibrosis. Although experimental studies have revealed targets to prevent fibrosis progression in rodents (20) (Table 2), the efficacy of most treatments has not been proven in humans. This is due to the need to perform serial liver biopsies to accurately assess changes in liver fibrosis, the necessity of long-term follow-up studies, and the fact that humans are probably less sensitive to hepatic antifibrotic therapies than rodents. The development of reliable noninvasive markers of liver fibrosis should have a positive impact on the design of clinical trials. The ideal antifibrotic therapy would be one that is liver-specific, well tolerated when administered for prolonged periods of time, and effective in attenuating excessive collagen deposition without affecting normal ECM synthesis.

The removal of the causative agent is the most effective intervention in the treatment of liver fibrosis. This strategy has been shown effective in most etiologies of chronic liver diseases $(19,122,124$, 125, S1, S2). For patients with cirrhosis and clinical complications, liver transplantation is currently the only curative approach (S5). Transplantation improves both survival and quality of life. However, in patients with HCV-induced cirrhosis, viral infection recurs after transplantation (S6), aggressive chronic hepatitis develops, and progression to cirrhosis is common.

Because inflammation precedes and promotes the progression of liver fibrosis, the use of antiinflammatory drugs has been proposed. Corticosteroids are only indicated for the treatment of hepatic fibrosis in patients with autoimmune hepatitis and acute alcoholic hepatitis (S1). Inhibition of the accumulation of activated HSCs by modulating either their activation and/or proliferation or promoting their apoptosis is another strategy. 
Antioxidants such as vitamin E, silymarin, phosphatidylcholine, and S-adenosyl-L-methionine inhibit HSC activation, protect hepatocytes from undergoing apoptosis, and attenuate experimental liver fibrosis (S7). Antioxidants exert beneficial effects in patients with alcohol-induced liver disease and NASH (S8, S9). Disrupting TGF- $\beta$ synthesis and/or signaling pathways prevents scar formation in experimental liver fibrosis (58). Moreover, administration of growth factors (e.g., IGF, hepatocyte growth factor, and cardiotrophin) or their delivery by gene therapy attenuates experimental liver fibrosis (S10, S11). However, these latter approaches have not been tested in humans and may favor cancer development. Substances that inhibit key signal transduction pathways involved in liver fibrogenesis also have the potential to treat liver fibrosis (20). They include pentoxifylline (phosphodiesterase inhibitor), amiloride $\left(\mathrm{Na}^{+} / \mathrm{H}^{+}\right.$pump inhibitor), and S-farnesylthiosalicylic acid (Ras antagonist). Ligands of PPAR $\alpha$ and/or PPAR $\gamma$ such as thiazolindiones exert beneficial effects in experimental liver fibrosis and in patients with NASH (S12, S13). The inhibition of the renin-angiotensin system is probably the most promising strategy in treating liver fibrosis. Renin-angiotensin inhibitors are widely used as antifibrotic agents in patients with chronic renal and cardiac diseases and appear to be safe when administered for prolonged periods of time (S14). Little information is available on the use of this approach in patients with chronic liver diseases. Preliminary pilot studies in patients with chronic hepatitis $\mathrm{C}$ and $\mathrm{NASH}$ suggest that renin-angiotensin blocking agents may have beneficial effects on fibrosis progression (S15). Transplanted patients receiving renin-angiotensin system inhibitors as antihypertensive therapy show less fibrosis progression than patients receiving other types of drugs (S16). However, this approach cannot be recommended in clinical practice until the results of ongoing clinical trials become available. The blockade of endothelin-1 type A receptors and the administration of vasodilators (prostaglandin E2 and nitric oxide donors) exert antifibrotic activity in rodents, yet the effects in humans are unknown (90). Different herbal compounds, many of them traditionally used in Asian countries to treat liver diseases, have been demonstrated to have antifibrotic effects (S17). They include Sho-saiko-to, glycyrrhizin, and savia miltiorbiza. An alternative approach is the inhibition of collagen production and/or the promotion of its degradation (20). Inhibitors of prolyl-4 hydroxylase and halofuginone prevent the development of experimental liver cirrhosis by inhibiting collagen synthesis. MMP-8 and urokinasetype plasminogen activator stimulate collagen degradation in vivo. The efficacy of these drugs in humans is unknown, and they may result in undesirable side effects. Finally, infusion of mesenchymal stem cells ameliorates experimentally induced fibrosis, which suggests a potential for this approach in the treatment of chronic liver diseases (S18, S19).

A limitation of the current antifibrotic approaches is that antifibrotic drugs are not efficiently taken up by activated HSCs and may produce unwanted side effects. Cell-specific delivery to HSCs could provide a solution to these problems. Promising preliminary results have been recently obtained using different carriers (e.g., cyclic peptides coupled to albumin recognizing collagen type VI receptor and/or PDGFR) (S20). Antifibrotic therapy may differ depending on the type of liver disease. In patients with chronic HCV infection, current antiviral treatments (pegylated IFN plus ribavirin) clear viral infection in more than half of the patients (S21). Sustained virological response is associated with an improvement in liver fibrosis (122). Patients with no sustained response may also experience improvement of liver fibrosis, which suggests that IFN- $\alpha$ has an intrinsic antifibrotic effect (S22). For nonresponder patients, the use of renin-angiotensin system inhibitors is a promising approach. Treatment of the metabolic syndrome in patients with chronic hepatitis $\mathrm{C}$ may also decrease fibrosis progression (S23). In patients with alcohol-induced liver disease, the most effective approach is alcohol abstinence (124). Antioxidants (e.g., S-adenosyl-L-methionine and phosphatidylcholine) and hepatocyte protectors (e.g., silymarin) slow down the progression of liver fibrosis and can improve survival (S24). For patients with autoimmune hepatitis, immunosuppressant therapy not only decreases inflammation but also exerts antifibrotic effects (S25). No antifibrotic therapy is available for patients with chronic cholestatic disorders (i.e., primary sclerosing cholangitis and PBC). Ursodeoxycholic acid improves biochemical tests in these patients, but its impact on fibrosis is not consistently proven (S26). In patients with NASH, weight loss and specific treatments of the metabolic syndrome can reduce fibrosis development (125). Recent reports have revealed than antioxidants and insulin sensitizers (e.g., thiazolindiones) may exert antifibrogenic effects in these patients (S27). Large clinical trials are needed to confirm these results.

\section{Future directions}

The translation of basic research into improved therapeutics for the management of patients with chronic liver diseases is still poor. The role of pluripotential stem cells in hepatic wound healing is one of the most promising fields. Perfusion of these cells may be a potential approach to promoting fibrosis resolution and liver regeneration. Approaches to removing fibrogenic cells are being evaluated, including development of drug delivery systems that target activated HSCs. Translational research should investigate the molecular mechanisms that cause fibrosis in different types of human liver diseases in order to identify new targets for therapy. In the clinical setting, the identity of the genetic determinants that influence fibrosis progression should be uncovered. Welldesigned large-scale epidemiological genetic studies are clearly required. Patients at a high risk of progression to cirrhosis should be identified. Developing simple and reliable noninvasive markers of hepatic fibrosis is an important goal in clinical hepatology and will facilitate the design of clinical trials. Most importantly, the efficacy of antifibrotic drugs known to attenuate experimental liver fibrosis should be tested in humans.

\section{Acknowledgments}

The authors' work is supported by grants from the NIH, the Ministerio de Ciencia y Tecnología de España, and the Instituto de Investigación Carlos III (SAF2002-03696 and BFI2002-01202).

Due to space constraints, a number of important references could not be included in this article. References S1-S27 are available online with this article; doi:10.1172/JCI200524282DS1.

Address correspondence to: David A. Brenner, Department of Medicine, Columbia University Medical Center, College of Physicians and Surgeons, 622 West 168th Street, PH 8E-105J, New York, New York 10032, USA. Phone: (212) 305-5838; Fax: (212) 305-8466; E-mail:dab2106@columbia.edu. 
1. Friedman, S.L. 2003. Liver fibrosis - from bench to bedside. J. Hepatol. 38(Suppl. 1):S38-S53.

2. Gines, P., Cardenas, A., Arroyo, V., and Rodes, J. 2004. Management of cirrhosis and ascites. N. Engl. J. Med. 350:1646-1654.

3. Popper, H., and Uenfriend, S. 1970 . Hepatic fibrosis. Correlation of biochemical and morphologic investigations. Am. J. Med. 49:707-721.

4. Schaffner, F., and Klion, F.M. 1968. Chronic hepatitis. Annu. Rev. Med. 19:25-38.

5. Albanis, E., and Friedman, S.L. 2001. Hepatic fibrosis. Pathogenesis and principles of therapy. Clin. Liver Dis. 5:315-334, v-vi.

6. Soyer, M.T., Ceballos, R., and Aldrete, J.S. 1976. Reversibility of severe hepatic damage caused by jejunoileal bypass after re-establishment of normal intestinal continuity. Surgery. 79:601-604.

7. Friedman, S.L., Roll, F.J., Boyles, J., and Bissell, D.M. 1985. Hepatic lipocytes: the principal collagen-producing cells of normal rat liver. Proc. Natl. Acad. Sci. U. S. A. 82:8681-8685.

8. Geerts, A. 2001. History, heterogeneity, developmental biology, and functions of quiescent hepatic stellate cells. Semin. Liver Dis. 21:311-335.

9. Friedman, S.L., et al. 1992. Isolated hepatic lipocytes and Kupffer cells from normal human liver: morphological and functional characteristics in primary culture. Hepatology. 15:234-243.

10. Otto, D.A., and Veech, R.L. 1980. Isolation of a lipocyte-rich fraction from rat liver nonparenchymal cells. Adv. Exp. Med. Biol. 132:509-517.

11. Rockey, D.C., Boyles, J.K., Gabbiani, G., and Friedman, S.L. 1992. Rat hepatic lipocytes express smooth muscle actin upon activation in vivo and in culture. J. Submicrosc. Cytol. Pathol. 24:193-203.

12. Pinzani, M., Gesualdo, L., Sabbah, G.M., and Abboud, H.E. 1989. Effects of platelet-derived growth factor and other polypeptide mitogens on DNA synthesis and growth of cultured rat liver fatstoring cells. J. Clin. Invest. 84:1786-1793.

13. Wasser, S., and Tan, C.E. 1999. Experimental models of hepatic fibrosis in the rat. Ann. Acad. Med. Singapore. 28:109-111.

14. Ramadori, G., and Saile, B. 2004. Portal tract fibrogenesis in the liver. Lab. Invest. 84:153-159.

15. Forbes, S.J., et al. 2004. A significant proportion of myofibroblasts are of bone marrow origin in human liver fibrosis. Gastroenterology. 126:955-963.

16. Poynard, T., et al. 2000. Natural history of HCV infection. Baillieres Best Pract. Res. Clin. Gastroenterol. 14:211-228.

17. Poynard, T., Bedossa, P., and Opolon, P. 1997. Natural history of liver fibrosis progression in patients with chronic hepatitis C. The OBSVIRC, METAVIR, CLINIVIR, and DOSVIRC groups. Lancet. 349:825-832.

18. Bataller, R., North, K.E., and Brenner, D.A. 2003. Genetic polymorphisms and the progression of liver fibrosis: a critical appraisal. Hepatology. 37:493-503.

19. Hammel, P., et al. 2001. Regression of liver fibrosis after biliary drainage in patients with chronic pancreatitis and stenosis of the common bile duct. N. Engl. J. Med. 344:418-423.

20. Bataller, R., and Brenner, D.A. 2001. Hepatic stellate cells as a target for the treatment of liver fibrosis. Semin. Liver Dis. 21:437-451.

21. Brunt, E.M. 2004. Nonalcoholic steatohepatitis. Semin. Liver Dis. 24:3-20.

22. Ludwig, J., Viggiano, T.R., McGill, D.B., and Oh, B.J. 1980. Nonalcoholic steatohepatitis: Mayo Clinic experiences with a hitherto unnamed disease. Mayo Clin. Proc. 55:434-438.

23. Davis, G.L., Albright, J.E., Cook, S.F., and Rosenberg, D.M. 2003. Projecting future complications of chronic hepatitis C in the United States. Liver Transpl. 9:331-338.

24. Berenguer, M., et al. 2003. Severe recurrent hepatitis
$\mathrm{C}$ after liver retransplantation for hepatitis $\mathrm{C}$ virusrelated graft cirrhosis. Liver Transpl. 9:228-235.

25. Afdhal, N.H., and Nunes, D. 2004. Evaluation of liver fibrosis: a concise review. Am. J. Gastroenterol. 99:1160-1174.

26. Thampanitchawong, P., and Piratvisuth, T. 1999. Liver biopsy: complications and risk factors. World J. Gastroenterol. 5:301-304.

27. Regev, A., et al. 2002. Sampling error and intraobserver variation in liver biopsy in patients with chronic HCV infection. Am. J. Gastroenterol. 97:2614-2618.

28. Imbert-Bismut, F., et al. 2001. Biochemical markers of liver fibrosis in patients with hepatitis C virus infection: a prospective study. Lancet. 357:1069-1075.

29. Forns, X., et al. 2002. Identification of chronic hepatitis $\mathrm{C}$ patients without hepatic fibrosis by a simple predictive model. Hepatology. 36:986-992.

30. Fontana, R.J., and Lok, A.S. 2002. Noninvasive monitoring of patients with chronic hepatitis C. Hepatology. 36:S57-S64.

31. Hirata, M., Akbar, S.M., Horiike, N., and Onji, M. 2001. Noninvasive diagnosis of the degree of hepatic fibrosis using ultrasonography in patients with chronic liver disease due to hepatitis $C$ virus. Eur. J. Clin. Invest. 31:528-535.

32. Callewaert, N., et al. 2004. Noninvasive diagnosis of liver cirrhosis using DNA sequencer-based total serum protein glycomics. Nat. Med. 10:429-434.

33. Pinzani, M. 1999. Liver fibrosis. Springer Semin. Immunopathol. 21:475-490.

34. Benyon, R.C., and Iredale, J.P. 2000. Is liver fibrosis reversible? Gut. 46:443-446.

35. Arthur, M.J. 2000. Fibrogenesis II. Metalloproteinases and their inhibitors in liver fibrosis. Am. J. Physiol. Gastrointest. Liver Physiol. 279:G245-G249.

36. Gabele, E., Brenner, D.A., and Rippe, R.A. 2003. Liver fibrosis: signals leading to the amplification of the fibrogenic hepatic stellate cell. Front. Biosci. 8:D69-D77.

37. Milani, S., et al. 1990. Procollagen expression by nonparenchymal rat liver cells in experimental biliary fibrosis. Gastroenterology. 98:175-184.

38. Marra, F. 1999. Hepatic stellate cells and the regulation of liver inflammation. J. Hepatol. 31:1120-1130.

39. Lindquist, J.N., Marzluff, W.F., and Stefanovic, B. 2000. Fibrogenesis. III. Posttranscriptional regulation of type I collagen. Am. J. Physiol. Gastrointest. Liver Physiol. 279:G471-G476.

40. Lindquist, J.N., Parsons, C.J., Stefanovic, B., and Brenner, D.A. 2004. Regulation of alpha1(I) collagen messenger RNA decay by interactions with alphaCP at the 3 '-untranslated region. J. Biol. Chem. 279:23822-23829.

41. Sato, M., Suzuki, S., and Senoo, H. 2003. Hepatic stellate cells: unique characteristics in cell biology and phenotype. Cell Struct. Funct. 28:105-112.

42. Oben, J.A., Yang, S., Lin, H., Ono, M., and Diehl, A.M. 2003. Norepinephrine and neuropeptide Y promote proliferation and collagen gene expression of hepatic myofibroblastic stellate cells. Biochem. Biophys. Res. Commun. 302:685-690.

43. Kinnman, N., and Housset, C. 2002. Peribiliary myofibroblasts in biliary type liver fibrosis. Front. Biosci. 7:d496-d503.

44. Magness, S.T., Bataller, R., Yang, L., and Brenner, D.A. 2004. A dual reporter gene transgenic mouse demonstrates heterognity in hepatic fibrogenic cell populations. Hepatology. 40:1151-1159.

45. Knittel, T., et al. 1999. Rat liver myofibroblasts and hepatic stellate cells: different cell populations of the fibroblast lineage with fibrogenic potential. Gastroenterology. 117:1205-1221.

46. Suskind, D.L., and Muench, M.O. 2004. Searching for common stem cells of the hepatic and hematopoietic systems in the human fetal liver: CD34+ cytokeratin $7 / 8+$ cells express markers for stellate cells. J. Hepatol. 40:261-268.

47. Phillips, R.J., et al. 2004. Circulating fibrocytes traffic to the lungs in response to CXCL12 and mediate fibrosis. J. Clin. Invest. 114:438-446. doi:10.1172/ JCI200420997.

48. Kalluri, R., and Neilson, E.G. 2003. Epithelialmesenchymal transition and its implications for fibrosis. J. Clin. Invest. 112:1776-1784. doi:10.1172/ JCI200320530.

49. Kmiec, Z. 2001. Cooperation of liver cells in health and disease. Adv. Anat. Embryol. Cell Biol. 161:III-XIII, 1-151.

50. Higuchi, H., and Gores, G.J. 2003. Mechanisms of liver injury: an overview. Curr. Mol. Med. 3:483-490.

51. Canbay, A., Friedman, S., and Gores, G.J. 2004. Apoptosis: the nexus of liver injury and fibrosis. Hepatology. 39:273-278.

52. Casini, A., et al. 1997. Neutrophil-derived superoxide anion induces lipid peroxidation and stimulates collagen synthesis in human hepatic stellate cells: role of nitric oxide. Hepatology. 25:361-367.

53. Vinas, O., et al. 2003. Human hepatic stellate cells show features of antigen-presenting cells and stimulate lymphocyte proliferation. Hepatology. 38:919-929.

54. Maher, J.J. 2001. Interactions between hepatic stellate cells and the immune system. Semin. Liver Dis. 21:417-426.

55. Shi, Z., Wakil, A.E., and Rockey, D.C. 1997. Strainspecific differences in mouse hepatic wound healing are mediated by divergent $T$ helper cytokine responses. Proc. Natl. Acad. Sci. U. S. A. 94:10663-10668.

56. Naito, M., Hasegawa, G., Ebe, Y., and Yamamoto, T. 2004. Differentiation and function of Kupffer cells. Med. Electron Microsc. 37:16-28.

57. Thurman, R.G. 1998. Alcoholic liver injury involves activation of Kupffer cells by endotoxin. Am.J. Physiol. 275:G605-G611.

58. Gressner, A.M., Weiskirchen, R., Breitkopf, K., and Dooley, S. 2002. Roles of TGF-beta in hepatic fibrosis. Front. Biosci. 7:d793-d807.

59. Olaso, E., et al. 2001. DDR2 receptor promotes MMP-2-mediated proliferation and invasion by hepatic stellate cells. J. Clin. Invest. 108:1369-1378. doi:10.1172/JCI200112373.

60. Takehara, T., et al. 2004. Hepatocyte-specific disruption of $\mathrm{Bcl}-\mathrm{xL}$ leads to continuous hepatocyte apoptosis and liver fibrotic responses. Gastroenterology. 127:1189-1197.

61. Canbay, A., et al. 2002. Fas enhances fibrogenesis in the bile duct ligated mouse: a link between apoptosis and fibrosis. Gastroenterology. 123:1323-1330.

62. Safadi, R., et al. 2004. Immune stimulation of hepatic fibrogenesis by CD8 cells and attenuation by transgenic interleukin-10 from hepatocytes. Gastroenterology. 127:870-882.

63. Sahai, A., Malladi, P., Melin-Aldana, H., Green, R.M., and Whitington, P.F. 2004. Upregulation of osteopontin expression is involved in the development of nonalcoholic steatohepatitis in a dietary murine model. Am. J. Physiol. Gastrointest. Liver Physiol. 287:G264-G273.

64. Yoshida, T., et al. 2004. SOCS1 is a suppressor of liver fibrosis and hepatitis-induced carcinogenesis. J. Exp. Med. 199:1701-1707.

65. Streetz, K.L., et al. 2003. Interleukin 6/gp130dependent pathways are protective during chronic liver diseases. Hepatology. 38:218-229.

66. Bataller, R., et al. 2003. NADPH oxidase signal transduces angiotensin II in hepatic stellate cells and is critical in hepatic fibrosis. J. Clin. Invest. 112:1383-1394. doi:10.1172/JCI200318212.

67. Oben, J.A., et al. 2004. Hepatic fibrogenesis requires sympathetic neurotransmitters. Gut. 53:438-445.

68. Yu, C., et al. 2003. Role of fibroblast growth factor type 1 and 2 in carbon tetrachloride-induced hepatic injury and fibrogenesis. Am. J. Pathol. 
163:1653-1662.

69. Kamada, Y., et al. 2003. Enhanced carbon tetrachloride-induced liver fibrosis in mice lacking adiponectin. Gastroenterology. 125:1796-1807.

70. Kanno, K., Tazuma, S., and Chayama, K. 2003. AT1A-deficient mice show less severe progression of liver fibrosis induced by $\mathrm{CCl}(4)$. Biochem. Biophys. Res. Commun. 308:177-183

71. Ueberham, E., et al. 2003. Conditional tetracyclineregulated expression of TGF-beta 1 in liver of transgenic mice leads to reversible intermediary fibrosis. Hepatology. 37:1067-1078.

72. Yoshiji, H., et al. 2002. Tissue inhibitor of metalloproteinases-1 attenuates spontaneous liver fibrosis resolution in the transgenic mouse. Hepatology. 36:850-860

73. Agarwal, D.P. 2001. Genetic polymorphisms of alcohol metabolizing enzymes. Pathol. Biol. 49:703-709.

74. Degoul, F., et al. 2001. Homozygosity for alanine in the mitochondrial targeting sequence of superoxide dismutase and risk for severe alcoholic liver disease. Gastroenterology. 120:1468-1474.

75. Jarvelainen, H.A., et al. 2001. Promoter polymorphism of the CD14 endotoxin receptor gene as a risk factor for alcoholic liver disease. Hepatology. 33:1148-1153.

76. Donaldson, P., et al. 2001. HLA and interleukin 1 gene polymorphisms in primary biliary cirrhosis: associations with disease progression and disease susceptibility. Gut. 48:397-402.

77. Corpechot, C., et al. 2001. Apolipoprotein E polymorphism, a marker of disease severity in primary biliary cirrhosis? J. Hepatol. 35:324-328.

78. Schuppan, D., Krebs, A., Bauer, M., and Hahn, E.G. 2003. Hepatitis C and liver fibrosis. Cell Death Differ. 10(Suppl. 1):S59-S67.

79. Powell, E.E., et al. 2000. Host genetic factors influence disease progression in chronic hepatitis C. Hepatology. 31:828-833.

80. Sasaki, K., et al. 2000. Mannose-binding lectin polymorphisms in patients with hepatitis $\mathrm{C}$ virus infection. Scand. J. Gastroenterol. 35:960-965.

81. Akuta, N., et al. 2001. Risk factors of hepatitis C virus-related liver cirrhosis in young adults: positive family history of liver disease and transporter associated with antigen processing $2(\mathrm{TAP} 2)^{*} 0201$ allele. J. Med. Virol. 64:109-116.

82. Martinelli, A.L., et al. 2000. Are haemochromatosis mutations related to the severity of liver disease in hepatitis C virus infection? Acta Haematol. 102:152-156.

83. Thorburn, D., et al. 2002. The role of iron and haemochromatosis gene mutations in the progression of liver disease in chronic hepatitis C. Gut. 50:248-252.

84. Day, C.P. 2004. The potential role of genes in nonalcoholic fatty liver disease. Clin. Liver Dis. 8:673-691, xi.

85. Marra, F. 2002. Chemokines in liver inflammation and fibrosis. Front. Biosci. 7:d1899-d1914.

86. Schwabe, R.F., Bataller, R., and Brenner, D.A. 2003. Human hepatic stellate cells express CCR5 and RANTES to induce proliferation and migration. Am. J. Physiol. Gastrointest. Liver Physiol. 285:G949-G958.

87. Shek, F.W., and Benyon, R.C. 2004. How can transforming growth factor beta be targeted usefully to combat liver fibrosis? Eur. J. Gastroenterol. Hepatol. 16:123-126.

88. Borkham-Kamphorst, E., Stoll, D., Gressner, A.M., and Weiskirchen, R. 2004. Antisense strategy against PDGF B-chain proves effective in preventing experimental liver fibrogenesis. Biochem. Biophys. Res. Commun. 321:413-423.

89. Williams, E.J., et al. 2001. Relaxin inhibits effective collagen deposition by cultured hepatic stellate cells and decreases rat liver fibrosis in vivo. Gut 49:577-583.

90. Cho, J.J., et al. 2000. An oral endothelin-A receptor antagonist blocks collagen synthesis and deposition in advanced rat liver fibrosis. Gastroenterology. 118:1169-1178.

91. Paizis, G., et al. 2002. Up-regulation of components of the renin-angiotensin system in the bile ductligated rat liver. Gastroenterology. 123:1667-1676.

92. Bataller, R., et al. 2003. Activated human hepatic stellate cells express the renin-angiotensin system and synthesize angiotensin II. Gastroenterology. 125:117-125

93. Jonsson, J.R., et al. 2001. Angiotensin-converting enzyme inhibition attenuates the progression of rat hepatic fibrosis. Gastroenterology. 121:148-155.

94. Paizis, G., et al. 2001. Effect of angiotensin II type 1 receptor blockade on experimental hepatic fibrogenesis. J. Hepatol. 35:376-385

95. Ramalho, L.N., et al. 2002. Effect of losartan, an angiotensin II antagonist, on secondary biliary cirrhosis. Hepatogastroenterology. 49:1499-1502.

96. Wei, H.S., et al. 2000. The regulatory role of AT receptor on activated HSCs in hepatic fibrogenesis: effects of RAS inhibitors on hepatic fibrosis induced by CCl(4). World J. Gastroenterol. 6:824-828.

97. Wei, H.S., et al. 2000. Effects of AT1 receptor antagonist, losartan, on rat hepatic fibrosis induced by $\mathrm{CCl}(4)$. World J. Gastroenterol. 6:540-545.

98. Tuncer, I., Ozbek, H., Ugras, S., and Bayram, I. 2003. Anti-fibrogenic effects of captopril and candesartan cilexetil on the hepatic fibrosis development in rat. The effect of AT1-R blocker on the hepatic fibrosis. Exp. Toxicol. Pathol. 55:159-166.

99. Bataller, R., et al. 2000. Angiotensin II induces contraction and proliferation of human hepatic stellate cells. Gastroenterology. 118:1149-1156.

100.Bataller, R., et al. 2003. Prolonged infusion of angiotensin II into normal rats induces stellate cell activation and proinflammatory events in liver. $A m$. J. Physiol. Gastrointest. Liver Physiol. 285:G642-G651.

101. Wheeler, M.D., et al. 2001. The role of Kupffer cell oxidant production in early ethanol-induced liver disease. Free Radic. Biol. Med. 31:1544-1549.

102.Kono, H., et al. 2000. NADPH oxidase-derived free radicals are key oxidants in alcohol-induced liver disease. J. Clin. Invest. 106:867-872.

103.Marra, F. 2002. Leptin and liver fibrosis: a matter of fat. Gastroenterology. 122:1529-1532.

104.Ikejima, K., et al. 2002. Leptin receptor-medi ated signaling regulates hepatic fibrogenesis and remodeling of extracellular matrix in the rat. Gastroenterology. 122:1399-1410.

105. Ortiz, V., Berenguer, M., Rayon, J.M., Carrasco, D., and Berenguer, J. 2002. Contribution of obesity to hepatitis C-related fibrosis progression. Am. J. Gastroenterol. 97:2408-2414.

106.Pinzani, M. 2002. PDGF and signal transduction in hepatic stellate cells. Front. Biosci. 7:d1720-d1726.

107. Marra, F., et al. 1999. Extracellular signal-regulated kinase activation differentially regulates plateletderived growth factor's actions in hepatic stellate cells, and is induced by in vivo liver injury in the rat. Hepatology. 30:951-958.

108.Schwabe, R.F., et al. 2004. Differential requirement for c-Jun NH2-terminal kinase in TNFalpha- and
Fas-mediated apoptosis in hepatocytes. FASEB J. 18:720-722.

109.Schwabe, R.F., Schnabl, B., Kweon, Y.O., and Brenner, D.A. 2001. CD40 activates NF-kappa $B$ and c-Jun $\mathrm{N}$-terminal kinase and enhances chemokine secretion on activated human hepatic stellate cells. J. Immunol. 166:6812-6819.

110.Schnabl, B., et al. 2001. The role of Smad3 in mediating mouse hepatic stellate cell activation. Hepatology. 34:89-100.

111.Dooley, S., et al. 2003. Smad7 prevents activation of hepatic stellate cells and liver fibrosis in rats. Gastroenterology. 125:178-191.

112. Marra, F., et al. 2000. Ligands of peroxisome proliferator-activated receptor gamma modulate profibrogenic and proinflammatory actions in hepatic stellate cells. Gastroenterology. 119:466-478.

113. Galli, A., et al. 2002. Antidiabetic thiazolidinediones inhibit collagen synthesis and hepatic stellate cell activation in vivo and in vitro. Gastroenterology. 122:1924-1940.

114.Boya, P., et al. 2001. Nuclear factor-kappa B in the liver of patients with chronic hepatitis $\mathrm{C}$ : decreased RelA expression is associated with enhanced fibrosis progression. Hepatology. 34:1041-1048.

115.Rippe, R.A., Schrum, L.W., Stefanovic, B., SolisHerruzo, J.A., and Brenner, D.A. 1999. NF-kappaB inhibits expression of the alpha1(I) collagen gene. DNA Cell Biol. 18:751-761.

116.Mann, D.A., and Smart, D.E. 2002. Transcriptional regulation of hepatic stellate cell activation. Gut. 50:891-896.

117.Paik, Y.H., et al. 2003. Toll-like receptor 4 mediates inflammatory signaling by bacterial lipopolysaccharide in human hepatic stellate cells. Hepatology. 37:1043-1055.

118.Canbay, A., et al. 2003. Cathepsin B inactivation attenuates hepatic injury and fibrosis during cholestasis. J. Clin. Invest. 112:152-159. doi:10.1172/ JCI200317740

119.Maher, J.J., Zia, S., and Tzagarakis, C. 1994. Acetaldehyde-induced stimulation of collagen synthesis and gene expression is dependent on conditions of cell culture: studies with rat lipocytes and fibroblasts. Alcohol Clin. Exp. Res. 18:403-409.

120.Bataller, R., Paik, Y.H., Lindquist, J.N., Lemasters, J.J., and Brenner, D.A. 2004. Hepatitis C virus core and nonstructural proteins induce fibrogenic effects in hepatic stellate cells. Gastroenterology. 126:529-540.

121. Wanless, I.R., and Shiota, K. 2004. The pathogenesis of nonalcoholic steatohepatitis and other fatty liver diseases: a four-step model including the role of lipid release and hepatic venular obstruction in the progression to cirrhosis. Semin. Liver Dis. 24:99-106.

122.Arthur, M.J. 2002. Reversibility of liver fibrosis and cirrhosis following treatment for hepatitis C. Gastroenterology. 122:1525-1528.

123.Issa, R., et al. 2004. Spontaneous recovery from micronodular cirrhosis: evidence for incomplete resolution associated with matrix cross-linking. Gastroenterology. 126:1795-1808.

124.Pares, A., Caballeria, J., Bruguera, M., Torres, M., and Rodes, J. 1986. Histological course of alcoholic hepatitis. Influence of abstinence, sex and extent of hepatic damage. J. Hepatol. 2:33-42.

125.Dixon, J.B., Bhathal, P.S., Hughes, N.R., and O'Brien, P.E. 2004. Nonalcoholic fatty liver disease: Improvement in liver histological analysis with weight loss. Hepatology. 39:1647-1654. 